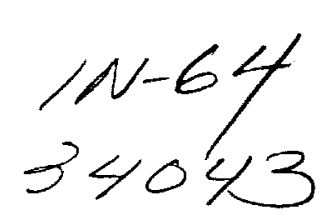

NASA Contractor Report 194992 $p-26$

ICASE Report No. 94-81
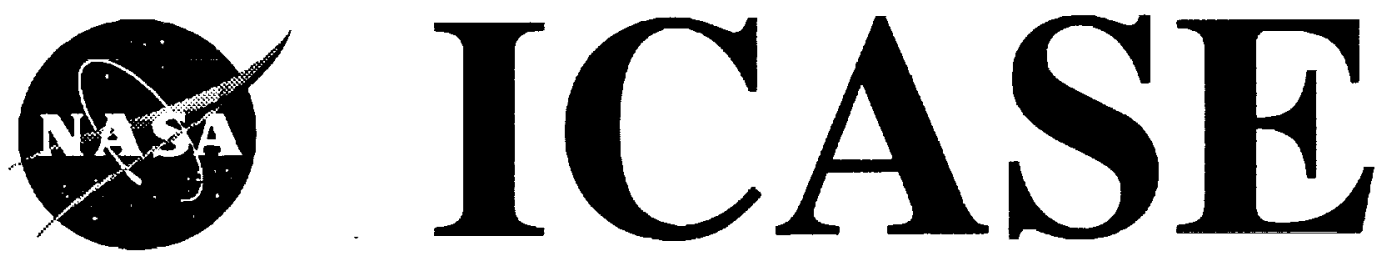

\title{
AN ANALYSIS OF SPECTRAL ENVELOPE-REDUCTION VIA QUADRATIC ASSIGNMENT PROBLEMS
}

\section{Alan George Alex Pothen}





\title{
AN ANALYSIS OF SPECTRAL ENVELOPE-REDUCTION VIA QUADRATIC ASSIGNMENT PROBLEMS *
}

\author{
ALAN GEORGE ${ }^{\dagger}$ AND ALEX POTHEN ${ }^{\ddagger}$
}

\begin{abstract}
A new spectral algorithm for reordering a sparse symmetric matrix to reduce its envelope size was described in [2]. The ordering is computed by associating a Laplacian matrix with the given matrix and then sorting the components of a specified eigenvector of the Laplacian. In this paper we provide an analysis of the spectral envelope reduction algorithm. We describe related 1- and 2-sum problems; the former is related to the envelope size, while the latter is related to an upper bound on the work involved in an envelope Cholesky factorization scheme. We formulate the latter two problems as quadratic assignment problems, and then study the 2-sum problem in more detail. We obtain lower bounds on the 2-sum by considering a projected quadratic assignment problem, and then show that finding a permutation matrix closest to an orthogonal matrix attaining one of the lower bounds justifies the spectral envelope reduction algorithm. The lower bound on the 2-sum is seen to be tight for reasonably "uniform" finite element meshes. We also obtain asymptotically tight lower bounds for the envelope size for certain classes of meshes.
\end{abstract}

* Written Sep. 1994.

$\dagger$ Department of Computer Science, University of Waterloo, Waterloo, Ontario, N2L 3G1 Canada (jageorgeesparse1.uvaterloo.ca). This author was supported by the Canadian Natural Sciences and Engineering Research Council under grant OGP0008111.

$\$$ Department of Computer Science, Old Dominion University, Norfolk, VA 23529-0162 and ICASE, NASA Langley Research Center, Hampton, VA 23681-0001 (pothen@cs.odu.edu, pothen@icase.edu). This author was supported by National Science Foundation grant CCR-9024954, by U. S. Department of Energy grants DE-FG02-91ER25095 and DE-FG05-94ER25216, by the National Aeronautics and Space Administration under NASA Contract NAS1-19480, and by the Canadian Natural Sciences and Engineering Research Council under grant OGP0008111. 

1. Introduction. A novel spectral algorithm to reduce the envelope of a sparse, symmetric matrix was described in a companion paper [2]. The algorithm associates a discrete Laplacian matrix with the given symmetric matrix, and then computes a reordering of the matrix by sorting the components of an eigenvector corresponding to the smallest nonzero Laplacian eigenvalue. The results in [2] show that the spectral algorithm can obtain significantly smaller envelope sizes compared to other currently used algorithms. All previous envelope-reduction algorithms (known to us), e.g., the reverse Cuthill-McKee (RCM) algorithm, the Gibbs-Poole-Stockmeyer (GPS) algorithm, the Gibbs-King (GK) algorithm, and the Sloan algorithm $[3,14,15,23,36]$, are combinatorial in nature, employing some variant of breadth-first-search to compute the ordering. In contrast, the spectral algorithm is an algebraic algorithm whose good envelope-reduction properties are somewhat intriguing and poorly understood.

In this paper we attempt to provide a raison d'être for the spectral envelope-reduction algorithm. We describe problems related to envelope-reduction called the 1- and 2-sum problems, and then formulate these latter problems as quadratic assignment problems (QAPs). We show that the QAP formulation of the 2-sum enables us to obtain lower bounds on the 2 -sum (and related envelope parameters) based on the Laplacian eigenvalues. The lower bounds seem to be quite tight for finite element problems whose mesh points are nearly all of the same degree. Further, we show that a closest permutation matrix to an orthogonal matrix that attains the lower bound is obtained by sorting the second Laplacian eigenvector components in monotonically increasing or decreasing order. This provides stronger justification for the spectral envelope-reducing algorithm than has been provided earlier.

The computational results in [2] indicate that the spectral envelope reduction algorithm is even more effective in reducing the work in an envelope factorization algorithm, due to the observed quadratic dependence of the factorization time on the envelope size. The analysis in this paper, in terms of the 2-sum problem, explains this phenomenon as well.

Although initially envelope-reducing orderings were developed for use in envelope schemes for sparse matrix factorization, these orderings have been used in the past few years in several other applications. The RCM ordering has been found to be an effective preordering in computing incomplete factorization preconditioners for preconditioned conjugate-gradient methods [4,6]. Envelope-reducing orderings have been used in frontal methods for sparse matrix factorization [7]. Such orderings have also been used in paralle ${ }^{-}$matrix-vector multiplication and tridiagonalization of sparse symmetric matrices.

The wider applicability of envelope-reducing orderings prompts us to take a fresh look at the reordering algorithms currently available, and to develop new ordering algorithms. Spectral envelope-reduction algorithms seem to be attractive in this context, since they

(i) compare favorably with existing algorithms in terms of the quality of the orderings [2], (ii) extend easily to problems with weights, e.g., finite element meshes arising from discretizations of anisotropic problems, and

(iii) are fairly easily parallelizable.

Spectral algorithms are usually, but not always, more expensive than the other algorithms currently available, but since the envelope-reduction problem requires only one eigenvector computation (to a few decimal digits of precision), we believe the costs are not impractically 
high. Improvements to reduce the costs are being looked into as well. We focus primarily on the class of finite element meshes arising from discretizations of partial differential equations. Our goals in this project are to develop efficient software implementing our algorithms, and to prove results about the quality of the orderings generated.

The projection approach for obtaining lower bounds of a QAP is due to Hadley, Rendl, and Wolkowicz [17], and this approach has been applied to the graph partitioning problem by the latter two authors [34]. In earlier work a spectral approach for the graph (matrix) partitioning problem has been employed to compute a spectral nested dissection ordering for sparse matrix factorization, for partitioning computations on finite element meshes on a distributed-memory multiprocessor $[19,31,32,35]$, and for load-balancing parallel computations [20]. The spectral approach has also been used to find a pseudo-peripheral node [16]. Juvan and Mohar [21, 22] have provided a theoretical study of the spectral algorithm for reducing $p$-sums, where $p=1,2$, and $\infty$, and Helmberg et al. [18] obtain spectral lower bounds on the bandwidth. A survey of some of these earlier results may be found in [29]. Paulino et al. [30] have also considered the use of spectral envelope-reduction for finite element problems.

The following is an outline of the rest of this paper. In Section 2 we describe various parameters of a matrix associated with its envelope, introduce the envelope size and envelope work minimization problems, and the related 1 - and 2 -sum problems. Let $\Delta$ denote the maximum number of offdiagonal nonzeros in a row or column of the given matrix (the maximum degree of a vertex in the adjacency graph of the matrix). We prove that the minimum 1-sum is bounded below by the minimum envelope size, and bounded above by $\Delta$ times the minimum envelope size. A similar result holds for the minimum 2 -sum and a bound on the work in an envelope Cholesky factorization. We compute upper and lower bounds for the envelope of a sparse symmetric matrix in terms of the eigenvalues of the Laplacian matrix in Section 3. The popular RCM ordering is obtained by reversing the Cuthill-McKee (CM) ordering, and usually the $\mathrm{RCM}$ ordering has smaller envelope size and work than the $\mathrm{CM}$ ordering. We prove that reversing an ordering can improve or impair the envelope size by at most a factor $\Delta$, and the envelope work by at most $\Delta^{2}$. In Section 4 , we formulate the 2 - and 1 -sum problems as quadratic assignment problems. We obtain lower and upper bounds for the 2-sum problem in terms of the eigenvalues of the Laplacian matrix in Section 5 by means of a projection approach that relaxes a permutation matrix to an orthogonal matrix with row and column sums equal to one. We also show how the lower bound may be strengthened by permitting diagonal perturbations to the Laplacian matrix. We justify the spectral envelope-reduction algorithm in Section 6 by proving that a closest permutation matrix to an orthogonal matrix attaining the lower bound for the 2-sum is obtained by permuting the second Laplacian eigenvector in monotonically increasing or decreasing order. In Section 7 we employ the lower bounds we have obtained to study the asymptotic growth of envelope size and an estimate of the envelope work for 2- and 3-dimensional finite element meshes of bounded degree. We show that graphs with good separators have small envelope parameters as well, by considering a modified nested dissection ordering. We present computational results in Section 8 to illustrate that the 2sums obtained by the spectral reordering algorithm can be close to optimal for finite element 
meshes whose nodes have almost equal degrees. We make some concluding remarks in Section 9. The Appendix contains some lower bounds for the more general $p$-sum problem, where $1 \leq p<\infty$.

\section{A menagerie of envelope problems.}

2.1. The envelope of a matrix. Let $A$ be an $n \times n$ symmetric matrix with elements $a_{i j}$, whose diagonal elements are nonzero. Various parameters of the matrix $A$ associated with its envelope are defined below.

We denote the column indices of the nonzeros in the lower triangular part of the $i$ th row by

$$
\operatorname{row}(i)=\left\{j: a_{i j} \neq 0 \text { and } 1 \leq j \leq i\right\} .
$$

For the $i$ th row of $A$ we define

$$
\begin{aligned}
& f_{i}(A)=\min \{j: j \in \operatorname{row}(i)\}, \quad \text { and } \\
& r_{i}(A)=i-f_{i}(A) .
\end{aligned}
$$

Here $f_{i}(A)$ is the column index of the first nonzero in the $i$ th row of $A$ (by our assumption of nonzero diagonals, $\left.1 \leq f_{i} \leq i\right)$, and the parameter $r_{i}(A)$ is the row-width of the $i$ th row of $A$. The bandwidth of $A$ is the maximum row-width

$$
b w(A)=\max \left\{r_{i}(A): i=1, \ldots, n\right\} .
$$

The envelope of $A$ is the set of column indices

$$
\operatorname{Env}(A)=\left\{(i, j): f_{i}(A) \leq j<i, i=1, \ldots, n\right\} .
$$

For each row, these indices lie in an interval beginning with the column index of the first nonzero element and ending with (but not including) the index of the diagonal nonzero element.

We denote the size of the envelope by $\operatorname{Esize}(A)=|\operatorname{Env}(A)|$. (This is also called the profile of $A$.) The work in the Cholesky factorization of $A$ that employs an envelope storage scheme can be bounded by $(1 / 2) \sum_{i=2}^{n} r_{i}\left(r_{i}+3\right)$. This bound can differ significantly from the actual work required on some problems, but for nearly uniform finite element problems without appendages, it is usually a good measure of the work. (The computational results on the work in an envelope factorization scheme in [2] indicate this is a good approximation as well.) Hence hereafter we will use

$$
W \text { bound }(A)=\sum_{i=1}^{n} r_{i}^{2}
$$

as a measure of the work in such a factorization.

A $3 \times 3$ 7-point grid and the nonzero structure of the corresponding matrix $A$ are shown in Figure 2.1. A ' - ' indicates a nonzero element, and $\mathrm{a}$ ' $*$ ' indicates a zero element that belongs to the lower triangle of the envelope in the matrix. The row-widths given in 

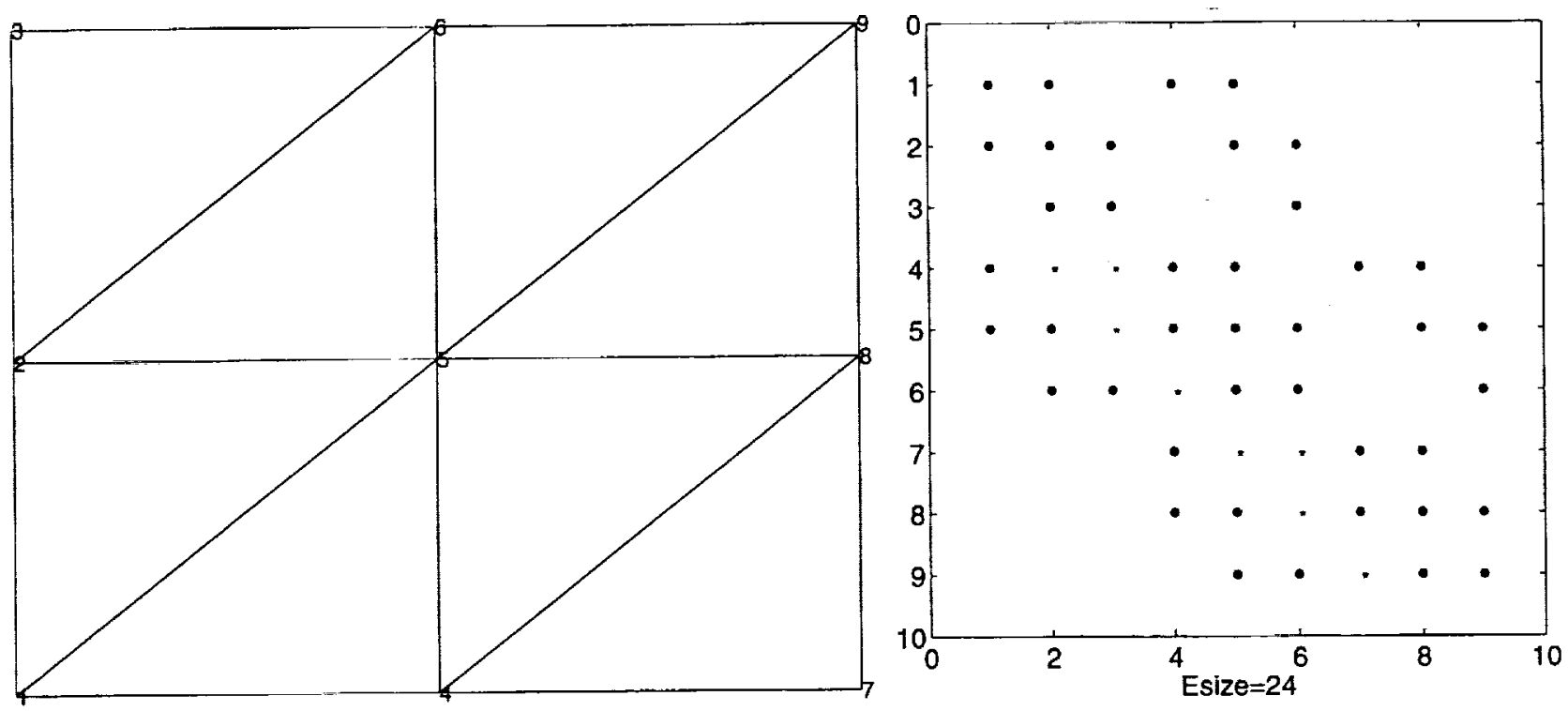

FIG. 2.1. An ordering of 7-point grid and the corresponding matrix. The lower triangle of the envelope is indicated by marking zeros within it by asterisks.

\begin{tabular}{c|ccccccccc}
$i$ & 1 & 2 & 3 & 4 & 5 & 6 & 7 & 8 & 9 \\
\hline$r_{i}$ & 0 & 1 & 1 & 3 & 4 & 4 & 3 & 4 & 4 \\
$c_{i}$ & 3 & 4 & 3 & 4 & 4 & 3 & 2 & 1 & 0
\end{tabular}

TABLE 2.1

Row-widths and column-widths of the matrix in Figure 1.

Table 2.1 are easily verified from the structure of the matrix. The envelope size is obtained by summing the row-widths, and is equal to 24 . (Column-widths $c_{i}$ are defined later in this section.)

The values of these parameters strongly depend on the choice of an ordering of the rows and columns. Hence we consider how these parameters vary over symmetrically permuted matrices $P^{T} A P$, where $P$ is a permutation matrix. We define $E s i z e_{\text {min }}(A)$, the minimum envelope size of $A$, to be the minimum envelope size among all permuted matrices $P^{T} A P$. The quantities $W$ bound $d_{\min }(A)$ and $b w_{\min }(A)$ are defined in similar fashion. Minimizing the envelope size and the bandwidth of a matrix are NP-complete problems [25], and minimizing the work bound is likely to be intractable as well. So one has to settle for heuristic orderings to reduce these quantities.

It is helpful to consider a 'column-oriented' expression for the envelope size for obtaining a lower bound on this quantity in Section 3. The width of a column $j$ of $A$ is the set of all row indices in the $j$ th column of the envelope of $A$. In other words,

$$
c_{j}(A)=\mid\left\{k: k>j \text {, and } \exists \ell \leq j \ni a_{k \ell} \neq 0\right\} \mid .
$$


(This is also called the $j$ th front-width.) It is then easily seen that the envelope size is

$$
\operatorname{Esize}(A)=\sum_{j=1}^{n} c_{j}
$$

The work in an envelope factorization scheme is given by

$$
\operatorname{Ework}(A)=(1 / 2) \sum_{j=1}^{n} c_{j}\left(c_{j}+3\right) .
$$

Hereafter, we will ignore the linear term in $c_{j}$ in computing the envelope work. The columnwidths of the matrix in Figure 2.1 are given in Table 2.1. These concepts and their interrelationships were considered earlier by Liu and Sherman [27], and are also discussed in the text books $[5,13]$.

The envelope parameters can also be defined with respect to the adjacency graph $G=$ $(V, E)$ of $A$. Denote $n b r(v)=\{v\} \cup a d j(v)$. In terms of the graph $G$ and an ordering $\alpha$ of its vertices, we can define

$$
\begin{aligned}
r(v, \alpha) & =\max \{\alpha(v)-\alpha(w): w \in n b r(v), \alpha(w) \leq \alpha(v)\} \\
\beta(G, \alpha) & =\max \{\alpha(v)-\alpha(w):(v, w) \in E\}
\end{aligned}
$$

Hence we can write the envelope size and work associated with an ordering $\alpha$ as

$$
\begin{aligned}
\operatorname{Esize}(G, \alpha) & =\sum_{v \in V} r(v)=\sum_{v \in V} \max \{\alpha(v)-\alpha(w): w \in n b r(v), \alpha(w) \leq \alpha(v)\} \\
\operatorname{Wbound}(G, \alpha) & =\sum_{v \in V} r(v)^{2}=\sum_{v \in V} \max \left\{(\alpha(v)-\alpha(w))^{2}: w \in n b r(v), \alpha(w) \leq \alpha(v)\right\} .
\end{aligned}
$$

The goal is to choose a vertex ordering $\alpha: V \mapsto\{1, \ldots, n\}$ to minimize one of the parameters described above. We denote by $\operatorname{Esize}_{\min }(G)\left(W_{b o u n d} d_{\min }(G)\right)$ the minimum value of $\operatorname{Esize}(G, \alpha)$ (Wbound $(G, \alpha)$ ) over all orderings $\alpha$. The reader can compute the envelope size of the numbered graph in Figure 2.1, using the definition given in this paragraph, to verify that $\operatorname{Esize}(G)=24$.

The $j$ th front-width has an especially nice interpretation if we consider the adjacency graph $G=(V, E)$ of $A$. Let the vertex corresponding to a column $j$ of $A$ be numbered $v_{j}$ so that $V=\left\{v_{1}, \ldots, v_{n}\right\}$, and define $V_{j}=\left\{v_{1}, \ldots, v_{j}\right\}$. Denote $\operatorname{adj}(X)=\left(\cup_{v \in X} \operatorname{adj}(v)\right) \backslash X$, for a subset of vertices $X$. Then $c_{j}(A)=\left|\operatorname{adj}\left(V_{j}\right)\right|$.

To illustrate the dependence of the envelope size on the ordering, we include in Figure 2.2 an ordering that leads to a smaller envelope size for the 7-point grid. Again, a ' $\bullet$ 'indicates a nonzero element, and a ' $*$ ' indicates a zero element that belongs to the lower triangle of the envelope in the matrix. This ordering by 'diagonals' yields the optimal envelope size for the 7-point grid [24].

2.2. 1- and 2-sum problems. It will be helpful to consider quantities related to the envelope size and envelope work, the 1-sum and the 2 -sum. We write the envelope size and 

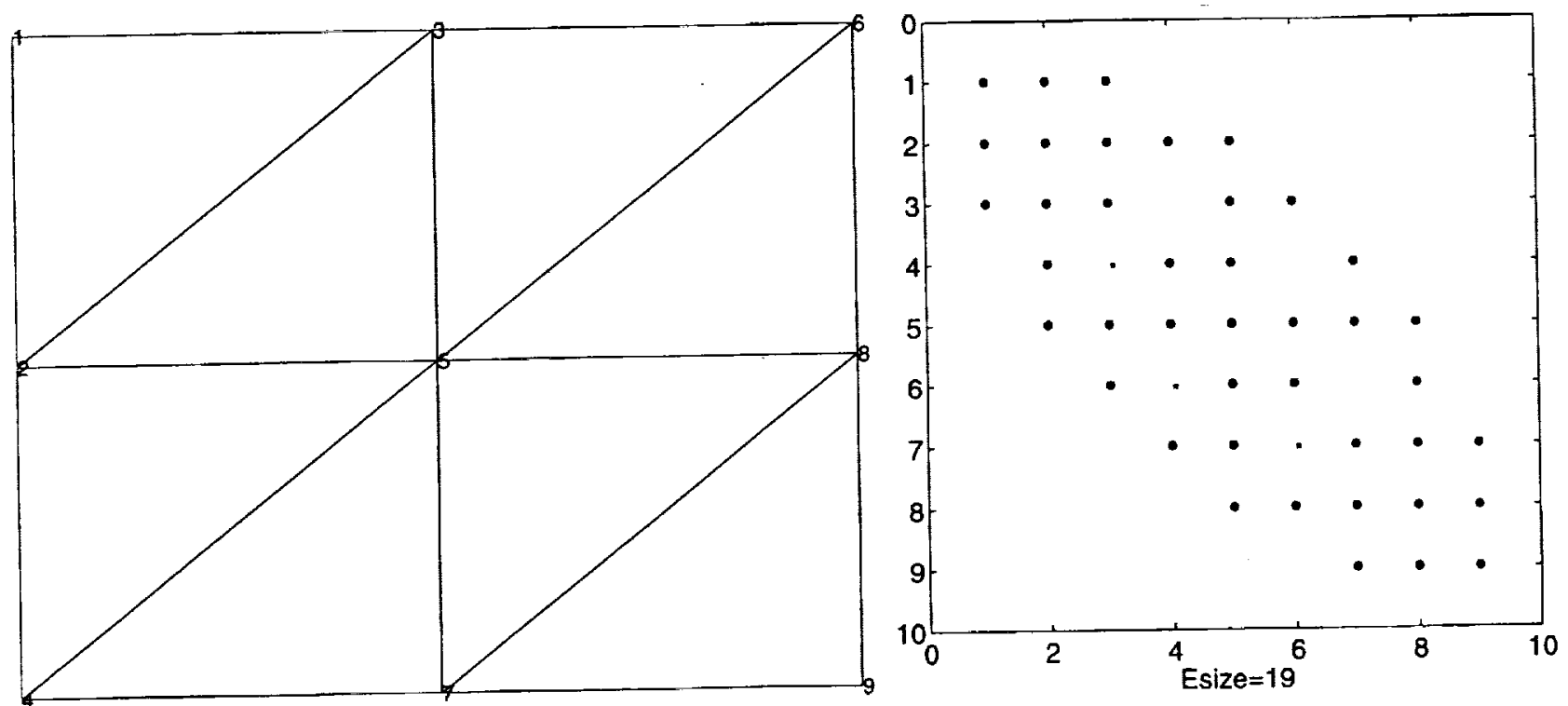

FIG. 2.2. Another ordering of a 7-point grid and the corresponding matrix. Again the lower triangle of the envelope is indicated by marking the zeros within it by asterisks.

1-sum, and the envelope work and the 2 -sum in a way that shows their relationships:

$$
\begin{aligned}
\text { Esize }(A) & =\sum_{i=1}^{n} \max _{j \in \operatorname{row}(i)}(i-j), \\
\sigma_{1}(A) & =\sum_{i=1}^{n} \sum_{j \in \operatorname{row}(i)}(i-j) ; \\
W \text { bound }(A) & =\sum_{i=1}^{n} \max _{j \in \operatorname{row}(i)}(i-j)^{2}, \\
\sigma_{2}^{2}(A) & =\sum_{i=1}^{n} \sum_{j \in \operatorname{row}(i)}(i-j)^{2} .
\end{aligned}
$$

The parameters $\sigma_{1, \min }(A)$ and $\sigma_{2, \min }^{2}(A)$ are the minimum values of these parameters over all permuted matrices $P^{T} A P$.

More generally, for real $1 \leq p<\infty$, we define the $p$-sum to be

$$
\sigma_{p}^{p}(A)=\sum_{i=1}^{n} \sum_{j \in \operatorname{row}(i)}(i-j)^{p}
$$

The 1-sum problem $(p=1)$ has also been called the optimal linear arrangement problem, and the limiting case $p=\infty$ corresponds to the bandwidth problem; both these problems are NP-complete.

We now consider the relationships between the envelope size problem and the 1-sum problem, and between the envelope work problem and the 2 -sum problem. Let $\Delta$ denote the maximum number of offdiagonal nonzeros in a row of $A$. (This is the maximum vertex degree in the adjacency graph of $A$.) 
THEOREM 2.1. The minimum values of the envelope size, envelope work in the Cholesky factorization, 1-sum, and 2-sum of a symmetric matrix $A$ are related by the following inequalities:

$$
\begin{aligned}
E_{\operatorname{size}_{\min }(A)} & \leq \sigma_{1, \min }(A) \leq \Delta E \operatorname{size}_{\min }(A) ; \\
\text { bbound }_{\min }(A) & \leq \sigma_{2, \min }^{2}(A) \leq \Delta W \text { bound }_{\min }(A) \\
\sigma_{2, \min }(A) & \leq \sigma_{1, \min }(A) \leq \sqrt{|E|} \sigma_{2, \min }(A) .
\end{aligned}
$$

Proof. We begin by proving (2.7). Our strategy will be to first prove the inequalities

$$
W \text { bound }(A) \leq \sigma_{2}^{2}(A) \leq \Delta W \text { bound }(A),
$$

and then to obtain the required result by considering two different permutations of $A$.

The bound $W$ bound $(A) \leq \sigma_{2}^{2}(A)$ is immediate from equations (2.4) and (2.5). If the inner sum in the latter equation is bounded from above by

$$
\Delta \max _{j \in \operatorname{row}(i)}(i-j)^{2},
$$

then we get $\Delta W$ bound $(A)$ as an upper bound on the 2-sum.

Now let $X_{1}$ be a permutation matrix such that $\widetilde{A_{1}} \equiv X_{1}^{T} A X_{1}$, and $W$ bound $\left(\widetilde{A_{1}}\right)=$ $W_{\text {bound }}$ min $(A)$. Then we have

$$
\sigma_{2, \min }^{2}(A) \leq \sigma_{2}^{2}\left(\widetilde{A_{1}}\right) \leq \Delta W \text { bound }\left(\widetilde{A_{1}}\right)=\Delta \text { Wbound }_{\min }(A) .
$$

Further, let $X_{2}$ be a permutation matrix such that $\widetilde{A_{2}} \equiv X_{2}^{T} A X_{2}$, and $\sigma_{2}^{2}\left(\widetilde{A_{2}}\right)=\sigma_{2, \min }^{2}(A)$. Again, we have

$$
\text { Wbound }_{\min }(A) \leq W \text { bound }\left(\widetilde{A_{2}}\right) \leq \sigma_{2}^{2}\left(\widetilde{A_{2}}\right)=\sigma_{2, \min }^{2}(A) .
$$

We obtain the result by putting the last two inequalities together.

We omit the proof of (2.6) since it can be obtained by a similar argument, and proceed to prove (2.8). The first inequality $\sigma_{2}(A) \leq \sigma_{1}(A)$ holds since the $p$-norm of any real vector is a decreasing function of $p$. The second inequality is also standard ${ }_{2=}$ since it bounds the 1-norm of a vector by means of its 2-norm. This result was obtained earlier by Juvan and Mohar [22]; we include its proof for completeness. Applying the Cauchy-Schwarz inequality to $\sigma_{1}^{2}(A)$ we have

$$
\begin{aligned}
& \left(\sum_{i=1}^{n} \sum_{j \in \operatorname{row}(i)}(i-j)\right)^{2} \\
& \leq\left(\sum_{i=1}^{n} \sum_{j \in \operatorname{row}(i)} 1\right)\left(\sum_{i=1}^{n} \sum_{j \in \operatorname{row}(i)}(i-j)^{2}\right)=|E| \sigma_{2}^{2}(A) .
\end{aligned}
$$

We obtain the result by considering two orderings that achieve the minimum 1- and 2-sums. 
3. Bounds for envelope size. In this section we present some bounds for the envelope size problem. We will require some background on the Laplacian matrix.

3.1. The Laplacian matrix. The Laplacian matrix $Q(G)$ of a graph $G$ is the $n \times n$ matrix $D-M$, where $D$ is the diagonal degree matrix and $M$ is the adjacency matrix of $G$. If $G$ is the adjacency graph of a symmetric matrix $A$, then we could define the Laplacian matrix $Q$ directly from $A$ :

$$
q_{i j}= \begin{cases}-1 & \text { if } i \neq j \text { and } a_{i j} \neq 0 \\ 0 & \text { if } i \neq j \text { and } a_{i j}=0 \\ \sum_{\substack{j=1 \\ j \neq i}}^{n}\left|q_{i j}\right| & \text { if } i=j .\end{cases}
$$

Note that

$$
\begin{aligned}
\underline{x}^{T} Q \underline{x} & =\underline{x}^{T} D \underline{x}-\underline{x}^{T} M \underline{x} \\
& =\sum_{\substack{j \leq i \\
a_{i j} \neq 0}}\left(x_{i}-x_{j}\right)^{2} .
\end{aligned}
$$

The eigenvalues of $Q(G)$ are the Laplacian eigenvalues of $G$, and we list them as $\lambda_{1}(Q) \leq$ $\lambda_{2}(Q) \leq \ldots \leq \lambda_{n}(Q)$. An eigenvector corresponding to $\lambda_{k}(Q)$ will be denoted by $\underline{x}_{k}$, and will be called a $k$ th eigenvector of $Q$. It is well-known that $Q$ is a singular $M$-matrix, and hence its eigenvalues are nonnegative. Thus $\lambda_{1}(Q)=0$, and the corresponding eigenvector is any nonzero constant vector $\underline{c}$. If $G$ is connected, then $Q$ is irreducible, and then $\lambda_{2}(Q)>0$; the smallest nonzero eigenvalues and the corresponding eigenvectors have important properties that make them useful in the solution of various partitioning and ordering problems. These properties were first investigated by Fiedler [8,9]; as discussed in Section 1, more recently several authors have studied their application to such problems.

3.2. Laplacian bounds for the envelope size. It would be helpful to work with the 'column-oriented' definition of the envelope size. Let the vertex corresponding to a column $j$ of $A$ be numbered $v_{j}$ in the adjacency graph so that $V=\left\{v_{1}, \ldots, v_{n}\right\}$, and let $V_{j}=\left\{v_{1}, \ldots, v_{j}\right\}$. Recall that the width of a column $j$ of $A$ is $c_{j}(A)=\left|\operatorname{adj}\left(V_{j}\right)\right|$, and that the envelope size is

$$
\operatorname{Esize}(A)=\sum_{j=1}^{n} c_{j}
$$

Recall also that $\Delta$ denotes the maximum degree of a vertex, and that given a set of vertices $S$, we denote by $\delta(S)$ the set of edges with one endpoint in $S$ and the other in $V \backslash S$.

We make use of the following elementary result, where the lower bound is due to Alon and Milman [1] and the upper bound is due to Juvan and Mohar [22].

LEMMA 3.1. Let $S \subset V$ be a subset of the vertices of a graph $G$. Then

$$
\lambda_{2}(Q) \frac{|S||V \backslash S|}{n} \leq|\delta(S)| \leq \lambda_{n}(Q) \frac{|S||V \backslash S|}{n} .
$$


THEOREM 3.2. The envelope size of a symmetric matrix $A$ can be bounded in terms of the eigenvalues of the associated Laplacian matrix as

$$
\frac{\lambda_{2}(Q)}{6 \Delta}\left(n^{2}-1\right) \leq \operatorname{Esize}(A) \leq \frac{\lambda_{n}(Q)}{6}\left(n^{2}-1\right) .
$$

Proof. From Lemma 3.1,

$$
\left|\delta\left(V_{j}\right)\right| \geq \frac{\lambda_{2}(Q)}{n} j(n-j) .
$$

Now $c_{j}(A)=\left|\operatorname{adj}\left(V_{j}\right)\right| \geq\left|\delta\left(V_{j}\right)\right| / \Delta$; substituting the lower bound for $\left|\delta\left(V_{j}\right)\right|$, and summing this latter expression over all $j$, we obtain the lower bound on the envelope size.

The upper bound is obtained by using the inequality $c_{j}(A) \leq\left|\delta\left(V_{j}\right)\right|$ with the upper bound in Lemma 3.1 .

Cuthill and McKee [3] proposed one of the earliest ordering algorithms for reducing the envelope size of a sparse matrix. George [12] discovered that reversing this ordering often leads to a significant reduction in envelope size and work. Since then the reverse-CuthillMcKee ( $\mathrm{RCM}$ ) ordering has become one of the most popular envelope size reducing orderings. However, we do not know of any published quantitative results on the improvement that may be expected by reversing an ordering, and here we present the first such result. For degreebounded finite element meshes, no asymptotic improvement is possible; the parameters are improved only by a constant factor.

THEOREM 3.3. Reversing the ordering of a sparse symmetric matrix $A$ can change (improve or impair) the envelope size by at most a factor $\Delta$, and the envelope work by at most $\Delta^{2}$, where $\Delta$ is the maximum number of offdiagonal nonzeros in a row (column) of $A$.

Proof. Let $v_{j}$ denote the vertex in the adjacency graph corresponding to the $j$ th column of $A$ (in the original ordering) so that the $j$ th column width $c_{j}(A)=\left|\operatorname{adj}\left(V_{j}\right)\right|$, where $V_{j}=$ $\left\{v_{1}, \ldots, v_{j}\right\}$. Let $\tilde{A}$ denote the permuted matrix obtained by reversing the column and row ordering of $A$. We have the inequality

$$
c_{j}(A)=\left|\operatorname{adj}\left(V_{j}\right)\right| \leq\left|\delta\left(V_{j}\right)\right| \leq \Delta\left|\operatorname{adj}\left(V \backslash V_{j}\right)\right|=\Delta c_{n-j}(\tilde{A}) .
$$

Since $\operatorname{Esize}(A)=\sum_{j=1}^{n} c_{j}(A)$, summing this inequality over $j$ from one to $n$, we obtain $\operatorname{Esize}(A) \leq \Delta \operatorname{Esize}(\widetilde{A})$. By symmetry, the inequality $\operatorname{Esize}(\widetilde{A}) \leq-\Delta E \operatorname{Esize}(A)$ holds as well.

The inequality on the envelope work follows by a similar argument from the equation $\operatorname{Ework}(A)=(1 / 2) \sum_{j=1}^{n} c_{j}^{2}$.

4. Quadratic assignment formulation of 2- and 1-sum problems. We formulate the 2- and 1-sum problems as quadratic assignment problems in this section.

4.1. The 2-sum problem. Let the vector $\underline{p}=\left(\begin{array}{llll}1 & 2 & \cdots & n\end{array}\right)^{T}$, and let $\underline{\alpha}$ be a permutation vector, i.e., a vector whose components form a permutation of $1, \ldots, n$. We may write $\underline{\alpha}=X \underline{p}$, where $X$ is a permutation matrix with elements

$$
x_{i j}=\left\{\begin{array}{ll}
1, & \text { if } j=\alpha(i) \\
0, & \text { otherwise }
\end{array} .\right.
$$


It is easily verified that the $(\alpha(i), \alpha(j))$ element of the permuted matrix $X^{T} A X$ is the element $a_{i j}$ of the unpermuted matrix $A$. Let $B=\underline{p} \underline{p}^{T}$; then $b_{i j}=i j$. We denote the set of all permutation vectors with $n$ components by $S_{n}$.

We write the 2-sum as a quadratic form involving the Laplacian matrix $Q$.

$$
\begin{aligned}
& \sigma_{2, \min }^{2}(A)=\min _{X} \sigma_{2}^{2}\left(X^{T} A X\right) \\
& =\min _{\underline{\alpha} \in S_{n}} \sum_{\substack{\alpha(j) \leq \alpha(i) \\
a_{\alpha(i), \alpha(j)} \neq 0}}(\alpha(i)-\alpha(j))^{2} \\
& =\min _{\underline{\alpha} \in S_{n}} \underline{\alpha}^{T} Q \underline{\alpha} \\
& =\min _{\underline{\alpha} \in S_{n}} \sum_{i=1}^{n} \sum_{j=1}^{n} q_{i j} \alpha(i) \alpha(j) .
\end{aligned}
$$

The transformation from the second to the third line makes use of (3.1).

This quadratic form can be expressed as a quadratic assignment problem by substituting $b_{\alpha(i), \alpha(j)}=\alpha(i) \alpha(j)$ :

$$
\min _{\underline{\alpha} \in S_{n}} \underline{\alpha}^{T} Q \underline{\alpha}=\min _{\underline{\alpha} \in S_{n}} \sum_{i=1}^{n} \sum_{j=1}^{n} q_{i j} b_{\alpha(i) \alpha(j)} .
$$

There is also a trace formulation of the QAP in which the variables are the elements of the permutation matrix $X$. We obtain this formulation by substituting $X \underline{p}$ for $\underline{\alpha}$. Thus

$$
\min _{\underline{\alpha} \in S_{n}} \underline{\alpha}^{T} Q \underline{\alpha}=\min _{X} \underline{p}^{T} X^{T} Q X \underline{p} .
$$

We may consider the last scalar expression as the trace of a $1 \times 1$ matrix, and then use the identity $\operatorname{tr} M N=\operatorname{tr} N M$ to rewrite the right-hand-side of the last displayed equation as

$$
\min _{X} \operatorname{tr} Q X \underline{p} \underline{p}^{T} X^{T} \equiv \min _{X} \operatorname{tr} Q X B X^{T}
$$

This is a quadratic assignment problem since it is a quadratic in the unknowns $x_{i j}$, which are the elements of the permutation matrix $X$. The fact that $B$ is a rank-one matrix leads to great simplifications and savings in the computation of good lower bounds for the 2-sum problem.

4.2. The 1-sum problem. Let $M$ be the adjacency matrix of a given symmetric matrix $A$ and let $S$ denote a 'distance matrix' with elements $s_{i j}=|i-j|$, both of order $n$. Then

$$
\begin{aligned}
& \sigma_{1, \min }(A)=\min _{X} \sigma_{1}\left(X^{T} A X\right) \\
& =\min _{\underline{\alpha} \in S_{n}} \sum_{\substack{\alpha(j) \leq \alpha(i) \\
m_{\alpha(i), \alpha(j) \neq 0}}} \alpha(i)-\alpha(j) \\
& =(1 / 2) \min _{\underline{\alpha} \in S_{n}} \sum_{i=1}^{n} \sum_{j=1}^{n} m_{i j} s_{\alpha(i), \alpha(j)} \\
& =(1 / 2) \min \operatorname{tr} M X S X^{T} .
\end{aligned}
$$


Unlike the 2-sum, the matrices involved in the QAP formulation of the 1-sum are both of rank $n$. Hence the bounds we obtain for this problem by this approach are considerably more involved.

\section{Eigenvalue bounds for the 2-sum problem.}

5.1. Orthogonal bounds. We have expressed the 2-sum problem as a QAP involving the Laplacian matrix $Q$ and the matrix $B=\underline{p} \underline{p}^{T}$, where $\underline{p}$ is an $n$-vector with $i$-th component equal to $i$. The trace formulation of the $\mathrm{QAP}$ is

$$
\min _{X} \operatorname{tr} Q X B X^{T}
$$

where $X$ ranges over the set of permutation matrices.

A technique for obtaining lower (upper) bounds for a QAP is to relax the requirement that the minimum (maximum) be attained over the class of permutation matrices. Denote the $n$-vector of all ones by $\underline{u}$. A matrix $X$ of order $n$ is a permutation matrix if and only if it satisfies the following three constraints:

$$
\begin{aligned}
X \underline{u} & =\underline{u}, \quad X^{T} \underline{u}=\underline{u} ; \\
X^{T} X & =I_{n} ; \\
x_{i j} & \geq 0, i, j=1, \ldots, n .
\end{aligned}
$$

The first of these, the stochasticity constraint, expresses the fact that each row or column of a permutation matrix has a single nonzero element with value one; the second states that a permutation matrix is orthonormal; and the third that its elements are non-negative. The simplest bounds for a QAP are obtained when we relax both the stochasticity and nonnegativity constraints, and insist only that $X$ be orthonormal. The following result is from [10]; see also [11].

THEOREM 5.1. Let the eigenvalues of a matrix be ordered

$$
\lambda_{1}(\cdot) \leq \lambda_{2}(\cdot) \cdots \leq \lambda_{n}(\cdot) .
$$

Then, as $X$ varies over the set of permutation matrices, the following upper and lower bounds hold:

$$
\sum_{i=1}^{n} \lambda_{i}(Q) \lambda_{n+1-i}(B) \leq \operatorname{tr} Q X B X^{T} \leq \sum_{i=1}^{n} \lambda_{i}(Q) \lambda_{i}(B) .
$$

The Laplacian matrix $Q$ has $\lambda_{1}(Q)=0$; also $\lambda_{i}(B)=0$, for $i=1, \ldots, n-1$, and $\lambda_{n}(B)=\underline{p}^{T} \underline{p}=(1 / 6) n(n+1)(2 n+1)$. Hence the lower bound in the theorem above is zero, and the upper bound is $(1 / 6) \lambda_{n}(Q) n(n+1)(2 n+1)$.

5.2. Projection bounds. Stronger bounds can be obtained by a projection technique described by Hadley, Rendl, and Wolkowicz [17]. The idea here is to satisfy the stochasticity constraints in addition to the orthonormality constraints, and relax only the non-negativity constraints. This technique involves projecting a permutation matrix $X$ into a subspace orthogonal to the stochasticity constraints (5.1) by means of an eigen-projection. 
From now on we normalize $\underline{u}:=(1 / \sqrt{n}) \underline{u}$, and let the $n \times n-1$ matrix $V$ be an orthonormal basis for the orthogonal complement of $\underline{u}$. By choice of $V$, we have $V^{T} \underline{u}=\underline{0}$, and $P=\left(\begin{array}{ll}\underline{u} & V\end{array}\right)$ is an orthonormal matrix of order $n$.

Observe that

$$
P^{T} X P=\left(\begin{array}{l}
\underline{u}^{T} \\
V^{T}
\end{array}\right) X\left(\begin{array}{ll}
\underline{u} & V
\end{array}\right)=\left(\begin{array}{ll}
\underline{u}^{T} X \underline{u} & \underline{u}^{T} X V \\
V^{T} X \underline{u} & V^{T} X V
\end{array}\right)=\left(\begin{array}{cc}
1 & \underline{0}^{T} \\
\underline{0} & Y
\end{array}\right),
$$

where $Y \equiv V^{T} X V$.

This suggests that we take

$$
\begin{aligned}
X & =P\left(\begin{array}{ll}
1 & \underline{0}^{T} \\
\underline{0} & Y
\end{array}\right) P^{T} \\
& =\underline{u} \underline{u}^{T}+V Y V^{T} .
\end{aligned}
$$

Note that with this choice, the stochasticity constraints $X \underline{u}=\underline{u}$, and $X^{T} \underline{u}=\underline{u}$ are satisfied. Furthermore, if $X$ is an orthonormal matrix of order $n$, then

$$
P^{T} X P=\left(\begin{array}{cc}
1 & \underline{0}^{T} \\
\underline{0} & Y
\end{array}\right)
$$

is orthonormal, and this implies that $Y$ is an orthonormal matrix of order $n-1$. Conversely, if $Y$ is orthonormal of order $n-1$, then the matrix $X$ obtained by the construction above is orthonormal of order $n$. The non-negativity constraint $X \geq 0$ becomes, from (5.4), $V Y V^{T} \geq-\underline{u} \underline{u}^{T}$. These facts will enable us to express the original QAP in terms of a projected QAP in the matrix of variables $Y$.

To obtain the projected QAP, we substitute the representation of $X$ from (5.4) into the objective function $\operatorname{tr} Q X B X^{T}$. Since $Q \underline{u}=\underline{0}$ by the construction of the Laplacian, terms of the form $Q \underline{u} \underline{u}^{T} \ldots$ vanish. Further,

$$
\operatorname{tr} Q V Y V^{T} B \underline{u} \underline{u}^{T}=\operatorname{tr} \underline{u}^{T} Q V Y V^{T} \underline{u},
$$

where we use the identity $\operatorname{tr} M N=\operatorname{tr} N M$ for an $n \times k$ matrix $M$ and a $k \times n$ matrix $N$. Again this term is zero since $\underline{u}^{T} Q=\underline{0}^{T}$. Hence the only nonzero term in the objective function is

$$
\begin{aligned}
& \operatorname{tr} Q V Y V^{T} B V Y^{T} V^{T} \\
& \quad=\operatorname{tr}\left(V^{T} Q V\right) Y\left(V^{T} B V\right) Y^{T} \\
& \quad=\operatorname{tr} \hat{Q} Y \hat{B} Y^{T}
\end{aligned}
$$

where $\widehat{M}=V M V^{T}$.

We have obtained the projected QAP in terms of the matrix $Y$ of order $n-1$, where the constraint that $X$ be a permutation matrix now imposes the constraints that $Y$ is orthonormal and that $V Y V^{T} \geq-\underline{u} \underline{u}^{T}$. We obtain lower and upper bounds in terms of the eigenvalues of the matrices $\widehat{Q}$ and $\widehat{B}$ by relaxing the non-negativity constraint again. 
THEOREM 5.2. The following upper and lower bounds hold for the 2-sum problem:

$$
(1 / 12) \lambda_{2}(Q)(n-1) n(n+1) \leq \sigma_{2}^{2}(A) \leq(1 / 12) \lambda_{n}(Q)(n-1) n(n+1) .
$$

Proof. If we apply the orthogonal bounds to the projected QAP, we get

$$
\sum_{i=1}^{n-1} \lambda_{i}(\hat{Q}) \lambda_{n-i}(\hat{B}) \leq \sigma_{2}^{2}(A) \leq \sum_{i=1}^{n-1} \lambda_{i}(\hat{Q}) \lambda_{i}(\hat{B})
$$

The vector $\underline{u}$ is the eigenvector of $Q$ corresponding to the zero eigenvalue, and hence eigenvectors corresponding to higher Laplacian eigenvalues are orthogonal to it. Thus any such eigenvector $\underline{x}_{j}$ can be expressed as $\underline{x}_{j}=V \underline{r}_{j}$. Substituting this last equation into the eigenvalue equation $Q \underline{x}_{j}=\lambda_{j}(Q) \underline{x}_{j}$, and pre-multiplying by $V^{T}$, we obtain $\widehat{Q}_{\underline{r}_{j}}=\lambda_{j}(Q) \underline{r}_{j}$. Hence for $i=2, \ldots, n$, we have $\lambda_{i}(Q)=\lambda_{i-1}(\hat{Q})$. Also, $\lambda_{n-1}(\widehat{B})=\underline{p}^{T} V V^{T} \underline{p}$, and all other eigenvalues are zero. Hence it remains to compute the largest eigenvalue of $\hat{B}$.

From the representation $I_{n}=P P^{T}=\underline{u} \underline{u}^{T}+V V^{T}$, we compute

$$
\begin{aligned}
& \underline{p}^{T} V V^{T} \underline{p}=\underline{p}^{T} \underline{p}-\left(\underline{p}^{T} \underline{u}\right)\left(\underline{u}^{T} \underline{p}\right) \\
& \quad=(1 / 6) n(n+1)(2 n+1)-(1 / 4) n(n+1)^{2}=(1 / 12)(n-1) n(n+1) .
\end{aligned}
$$

We get the result by substituting these eigenvalues into the bounds for the 2-sum.

For justifying the spectral algorithm for minimizing the 2-sum, we observe that the lower bound is attained by the matrix $X_{0}=\underline{u} \underline{u}^{T}+V R S^{T} V^{T}$, where $R(S)$ is a matrix of eigenvectors of $\hat{Q}(\hat{B})$, and the eigenvectors correspond to the eigenvalues of $\hat{Q}(\hat{B})$ in increasing (decreasing) order.

The result given above has been obtained by Juvan and Mohar [22] without using a QAP formulation of the 2-sum. We have included this proof for two reasons: First, in the next subsection, we show how the lower bound may be strengthened by diagonal perturbations of the Laplacian. Second, in the following section, we consider the problem of finding a permutation matrix closest to the orthogonal matrix attaining the lower bound.

5.3. Diagonal perturbations. The lower bound for the 2-sum can be further improved by perturbing the Laplacian matrix $Q$ by a diagonal matrix $\operatorname{Diag}(\underline{d})$, where $\underline{d}$ is an $n$-vector, and then using an optimization routine to maximize the smathest eigenvalue of the perturbed matrix.

Choosing the elements of $\underline{d}$ such that its elements sum to zero, i.e., $\underline{u}^{T} \underline{d}=0$, simplifies the bounds we obtain, and hence we make this assumption in this subsection. We begin by denoting $Q(d)=Q+\operatorname{Diag}(\underline{d})$, and expressing

$$
f(X) \equiv \operatorname{tr} Q X B X^{T}=\operatorname{tr} Q(d) X B X^{T}-\operatorname{tr} \operatorname{Diag}(\underline{d}) X B X^{T} .
$$

The second term can be written as a linear assignment problem (LAP) since one of the matrices involved is diagonal. Let the permutation vector $\underline{\alpha}=X \underline{p}$, and let $\underline{d}_{B}$ denote the $n$-vector formed from the diagonal elements of $B$.

$$
\operatorname{tr} \operatorname{Diag}(\underline{d}) X B X^{T}=\sum_{i=1}^{n} d_{i} b_{\alpha(i), \alpha(i)}=\operatorname{tr} \underline{d}_{B}{ }^{T} X^{T} .
$$


We now proceed, as in the previous subsection, to obtain projected bounds for the quadratic term, and thus for $f(X)$. Note that $Q(d) \underline{u}=(1 / \sqrt{n}) \underline{d}$ since $Q \underline{u}=\underline{0}$; and $\underline{u}^{T} Q(d) \underline{u}=0$ since the elements of $\underline{d}$ sum to zero. We shall write $B \underline{u}=(1 / \sqrt{n}) \underline{r}(B)$ to denote the row-sum of the elements of $B$.

With notation as in the previous subsection, we substitute $X=\underline{u} \underline{u}^{T}+V Y V^{T}$ in the quadratic term in $f(X)$. The first term $\operatorname{tr} Q(d) \underline{u}^{T} B \underline{u}^{T} \underline{u}^{T}=\operatorname{tr} \underline{u}^{T} Q(d) \underline{u} \underline{u}^{T} B \underline{u}=0$. The second and third terms are equal, and their sum can be transformed as follows:

$$
\begin{aligned}
2 & \operatorname{tr} Q(d) V Y V^{T} B \underline{u} \underline{u}^{T}=2 \operatorname{tr} \underline{u}^{T} Q(d) V Y V^{T} B \underline{u} \\
& =(2 / n) \operatorname{tr} \underline{d}^{T} V Y V^{T} \underline{r}(B)=(2 / n) \operatorname{tr} V^{T} \underline{r}(B) \underline{d}^{T} V Y \\
& =(2 / n) \operatorname{tr} Y^{T} V^{T} \underline{d} \underline{r}(B)^{T} V=(2 / n) \operatorname{tr} \underline{d} \underline{r}(B)^{T} V Y^{T} V^{T} .
\end{aligned}
$$

Note that this term is linear in the projected variables $Y$, and we shall find it convenient to express it in terms of $X$ by the substitution $X^{T}-\underline{u} \underline{u}^{T}=V Y^{T} V^{T}$. Thus

$$
(2 / n) \operatorname{tr} \underline{d} \underline{r}(B)^{T} V Y^{T} V^{T}=(2 / n) \operatorname{tr} \underline{d} \underline{r}(B)^{T}\left(X^{T}-\underline{u} \underline{u}^{T}\right)=(2 / n) \operatorname{tr} \underline{d} \underline{r}(B)^{T} X^{T},
$$

since the second term is equal to $\operatorname{tr} \underline{u}^{T} \underline{d} \underline{r}(B)^{T} \underline{u}$, which is zero by the choice of $\underline{d}$.

Finally, the fourth term becomes $\operatorname{tr} \hat{Q}(d) Y \hat{B} Y^{T}$, where $\hat{Q}(d)=V^{T} Q(d) V$, and as before $\widehat{B}=V^{T} B V$.

Putting it all together, we obtain

$$
f(X)=\operatorname{tr} \hat{Q}(d) Y \hat{B} Y^{T}+\operatorname{tr}\left((2 / n) V^{T} \underline{d} \underline{r}(B)^{T} X^{T}-\underline{d} \underline{d}_{B}{ }^{T} X^{T}\right) .
$$

Observe that the first term is quadratic in the projected variables $Y$, and the remaining terms are linear in the original variables $X$. Our lower bound for the 2 -sum shall be obtained by minimizing the quadratic and linear terms separately.

We can simplify the linear assignment problem by noting that $B=\underline{p} \underline{p}^{T}$. Thus $r_{B, i}=$ $i \sum_{j=1}^{n} j=(1 / 2) n(n+1) i$, and hence $(2 / n) \underline{r}(B)=(n+1) \underline{p}$. Further, $\underline{d}_{B}=s q(\underline{p})$, the vector with $i$ th component equal to $i^{2}$. Hence the final expression for the linear assignment problem is

$$
\operatorname{tr} \underline{d}\left((n+1) \underline{p}^{T}-s q(\underline{p})^{T}\right) X^{T} .
$$

Let $L$ denote the minimum value of this problem (over the set of permutation matrices $\mathrm{X}$, for a given $\underline{d}$ ), which can be computed as the solution of a transportation problem.

The eigenvalues of $\widehat{B}$ can be computed as in the previous subsection. We may choose $\underline{d}$ to maximize the smallest eigenvalue of the matrix $\hat{Q}(d)$. Thus this discussion leads to the following result.

THEOREM 5.3. The minimum 2-sum of a symmetric matrix $A$ can be bounded as

$$
\sigma_{2, \min }^{2}(A) \geq \max _{\underline{d}}(1 / 12) \lambda_{\mathbf{1}}(\hat{Q}(d))(n-1) n(n+1)+L,
$$

where the components of the vector $\underline{d}$ sum to zero. 
6. Computing an approximate solution from the lower bound. Consider the problem of finding a permutation matrix $Z$ "closest" to an orthogonal matrix $X_{0}$ that attains the lower bound in Theorem 5.2. We show in this section that sorting the second Laplacian eigenvector components in non-increasing (also non-decreasing) order yields a permutation matrix that solves a linear approximation to the problem. This justifies the spectral approach for minimizing the 2-sum.

Since $X_{0}$ attains the lower bound in Theorem 5.2, $X_{0}=\underline{u} \underline{u}^{T}+V R S^{T} V^{T}$, where $R(S)$ is a matrix of eigenvectors of $\hat{Q}(\hat{B})$ corresponding to the eigenvalues of $\hat{Q}(\widehat{B})$ in increasing (decreasing) order. We begin with a preliminary discussion of some properties of the matrix $X_{0}$ and the eigenvectors of $Q$. For $j=1, \ldots, n-1$, let the $j$ th column of $R$ be denoted by $\underline{r}_{j}$, and similarly let $\underline{s}_{j}$ denote the $j$ th column of $S$. Then $\underline{s}_{1}=V^{T} \underline{p}$, and for $j=2, \ldots$, $n-1$, the vector $\underline{s}_{j}$ is orthogonal to $V^{T} \underline{p}$, i.e.,

$$
\underline{s}_{j}^{T} V^{T} \underline{p}=0 \text {. }
$$

Recall from the previous section that a second Laplacian eigenvector $\underline{x}_{2}=V \underline{r}_{1}$.

Now we can formulate the "closest" permutation matrix problem more precisely. The minimum 2-sum problem may be written as

$$
\min _{Z}\left\|(Q+\alpha I)^{1 / 2} Z \underline{p}\right\|_{2}^{2} .
$$

We have chosen a positive shift $\alpha$ to make the shifted matrix positive definite and hence to obtain a weighted norm by making the square root nonsingular. It can be verified that the shift has no effect on the minimizer since it adds only a constant term to the objective function.

We substitute $Z=X_{0}+\left(Z-X_{0}\right)$ and expand the 2 -sum about $X_{0}$ to obtain

$$
\left\|(Q+\alpha I)^{1 / 2} Z \underline{p}\right\|_{2}{ }^{2}=
$$

$$
\left\|(Q+\alpha I)^{1 / 2} X_{0} \underline{p}\right\|_{2}{ }^{2}+2 \operatorname{tr} \underline{p}^{T}\left(Z-X_{0}\right)^{T}(Q+\alpha I) X_{0} \underline{p}+\left\|(Q+\alpha I)^{1 / 2}\left(Z-X_{0}\right) \underline{p}\right\|_{2}{ }^{2} .
$$

The first term on the right-hand-side is a constant since $X$ is a given orthogonal matrix; the third term is a quadratic in the difference $\left(Z-X_{0}\right)$ and hence we neglect it to obtain a linear approximation. It follows that we can choose a permutation matrix $Z$ close to $X_{0}$ to approximately minimize the 2 -sum by solving

$$
\min _{Z} \operatorname{tr} \underline{p}^{T} Z^{T}(Q+\alpha I) X_{0} \underline{p}=\min _{Z} \operatorname{tr}(Q+\alpha I) X_{0} B Z^{T} .
$$

Substituting for $X_{0}$ from (5.4) in this linear assignment problem and noting that $Q \underline{u}=\underline{0}$, we find

$$
\begin{gathered}
\min _{Z} \operatorname{tr}(Q+\alpha I) X_{0} B Z^{T}=\min _{Z} \operatorname{tr}(Q+\alpha I)\left(\underline{u}^{T}+V R S^{T} V^{T}\right) B Z^{T} \\
=\min _{Z} \operatorname{tr} Q V R S^{T} V^{T} B Z^{T}+\alpha \operatorname{tr} \underline{u}^{T} B Z^{T}+\alpha \operatorname{tr} V R S^{T} V^{T} B Z^{T} .
\end{gathered}
$$


The second term on the right-hand-side is a constant since

$$
\begin{aligned}
\operatorname{tr} & \underline{u} \underline{u}^{T} B Z^{T} \\
& =\left(\underline{u}^{T} \underline{p}\right) \operatorname{tr} \underline{u} \underline{p}^{T} Z^{T} \\
& =\left(\underline{u}^{T} \underline{p}\right) \operatorname{tr} Z^{T} \underline{u} \underline{p}^{T} \\
& =\left(\underline{u}^{T} \underline{p}\right) \operatorname{tr} \underline{u} \underline{p}^{T} \\
& =\left(\underline{u}^{T} \underline{p}\right)^{2} .
\end{aligned}
$$

Here we have substituted $Z^{T} \underline{u}=\underline{u}$ from (5.1). We proceed to simplify the first term in (6.4), which is

$$
\operatorname{tr} Q V R S^{T} B Z^{T}=\operatorname{tr} Q V\left(\sum_{j=1}^{n-1} \underline{r}_{j} \underline{s}_{j}^{T}\right) V^{T} \underline{p} \underline{p}^{T} Z^{T} .
$$

From (6.1) we find that $\underline{s}_{j}^{T} V^{T} \underline{p}=0$, for $j=2, \ldots, n-1$, and hence only the first term in the sum survives. Noting that $\underline{s}_{1}=V^{T} \underline{p}$, and $V \underline{r}_{1}=\underline{x}_{2}$, this term becomes

$$
\operatorname{tr} Q \underline{x}_{2} \underline{p}^{T} V V^{T} \underline{\underline{p}} \underline{p}^{T} Z^{T}=\lambda_{2}(Q)\left(\underline{p}^{T} V V^{T} \underline{p}\right) \operatorname{tr} \underline{x}_{2} \underline{p}^{T} Z^{T} .
$$

The third term in (6.4) can be simplified in like manner, and hence ignoring the constant second term, this equation becomes

$$
\left(\lambda_{2}(Q)+\alpha\right)\left(\underline{p}^{T} V V^{T} \underline{p}\right) \min _{Z} \operatorname{tr} \underline{x}_{2} \underline{p}^{T} Z^{T} .
$$

Hence we are required to choose a permutation matrix $Z$ to minimize $\operatorname{tr} \underline{x}_{2} \underline{p}^{T} Z^{T}=$ $\operatorname{tr} Z^{T} \underline{x}_{2} \underline{p}^{T}$. The solution to this problem is to choose $Z$ to correspond to a permutation of the components of $\underline{x}_{2}$ in non-increasing order, since the components of the vector $\underline{p}$ are in increasing order. Note that $-\underline{x}_{2}$ is also an eigenvector of the Laplacian matrix, and since the positive or negative signs of the components are chosen arbitrarily, sorting the eigenvector components into non-decreasing order also gives a permutation matrix $Z$ closest to the orthogonal matrix $X_{0}$.

Similar techniques can be used to show that if one is interested in maximizing the 2-sum, then a closest permutation matrix to the orthogonal matrix that attains the upper bound in Theorem 5.2 is approximated by sorting the components of the Laplacian eigenvector $\underline{x}_{n}$ (corresponding to the largest eigenvalue $\lambda_{n}(Q)$ ) in non-decreasing (non-increasing) order.

7. Implications. In this section we consider the implications of the spectral lower bounds that we have obtained. We denote the eigenvalues $\lambda_{2}(Q)$ by $\lambda_{2}$ and $\lambda_{n}(Q)$ by $\lambda_{n}$ for the sake of brevity in this section. We make use of the following result whose proof may be found in [33]. Let $\left\{G_{n}\right\}$ represent a family of graphs with parameter $n$ representing the number of vertices, and let $G$ be a graph on $n$ vertices from this family. Then $G$ has an $f(n)$-separator $S$ if $|S|=\Theta(f(n))$, and $S$ separates $G \backslash S$ into two parts $A, B$ with $h n \leq|A|,|B| \leq(1-h) n$, where $0<h<1$. (Thus $A$ and $B$ have $\Theta(n)$ vertices.) For instance, planar graphs have $\sqrt{n}$-separators. 
THEOREM 7.1. If the family $\left\{G_{n}\right\}$ has $f(n)$-separators, then

$$
\lambda_{2} \leq K \Delta(n) \frac{f(n)}{n},
$$

where $K$ is a constant independent of $n$, and $\Delta(n)$ is the maximum degree of any vertex in G.

Results of this nature, involving separator properties of graphs, provide the tightest known upper bounds for the second eigenvalue. Other results [8] that bound $\lambda_{2}$ in terms of the edge or vertex connectivity lead to an upper bound equal to at least one.

We can use information about the asymptotic behavior of the second eigenvalues together with the bounds we have obtained to predict the behavior of envelope parameters. For the envelope size, we make use of Theorem 3.2; for the estimate of the envelope work $W$ bound $(A)$, we will need to combine Theorem 5.2 and Theorem 2.1 to obtain the following result.

THEOREM 7.2. The estimate of the minimum work in an envelope factorization scheme of a symmetric positive definite matrix $A$ is bounded by

$$
\text { Wbound }_{\min }(A) \geq \frac{1}{12 \Delta} \lambda_{2}(Q)(n-1) n(n+1) .
$$

(Note that we have chosen to use the simpler bounds in Theorem 5.2 rather than the bound from Theorem 5.3.)

The bound on envelope size is tight for rather dense graphs and matrices. For instance, the full matrix (the complete graph) has $\lambda_{2}=\Delta+1=n$, and hence Esize $\min (A)=\Theta\left(n^{2}\right)$. Similarly we find that the bound on the envelope work $W$ bound $_{\min }(A)=\Theta\left(n^{3}\right)$. Moreover, the predicted lower bound is within a factor of three of the envelope size. These bounds are also asymptotically tight for random graphs where each possible edge is present in the graph with a fixed probability $p$, since the second Laplacian eigenvalue satisfies [21]

$$
\lambda_{2}=p n-\Theta\left([p(1-p) n \log n]^{1 / 2}\right) .
$$

We are interested in understanding the implications of these bounds for finite element meshes in two and three dimensions, with the maximum degree $\Delta$ bounded independent of $n$. For planar meshes, since $f(n)=\mathcal{O}\left(n^{1 / 2}\right)$, we have that $\lambda_{2}=\mathcal{O}\left(n^{-1 / 2}\right)$. Note that 'well-shaped' two-dimensional finite element meshes have $\lambda_{2}=\Theta\left(h^{2}\right)=\Theta\left(n^{-1}\right)$, where $h$ is the (uniform) distance between two successive points of the mesh along the $x$ - or $y$-axis. For three-dimensional meshes that possess $n^{2 / 3}$ separators, we have $\lambda_{2}=\mathcal{O}\left(n^{-1 / 3}\right)$. Again for 'good' three-dimensional meshes, we have $\lambda_{2}=\Theta\left(h^{2}\right)=\Theta\left(n^{-2 / 3}\right)$.

Plugging in the bounds above for $\lambda_{2}$ into the envelope size and envelope work bound, we find that for planar meshes that have $\lambda_{2}=\Theta\left(n^{-1 / 2}\right)$, the envelope size $E$ size $_{\min }(A)=$ $\Omega\left(n^{3 / 2}\right)$, and the work bound is $W$ bound $d_{\min }(A)=\Omega\left(n^{5 / 2}\right)$. For planar meshes with $\lambda_{2}=$ $\Theta\left(n^{-1}\right)$, we obtain the lower bounds $E_{\text {size }}$ min $(A)=\Omega(n)$, and $W_{\text {bound }}(A)=\Omega\left(n^{2}\right)$. For three-dimensional meshes that have $\lambda_{2}=\Theta\left(n^{-1 / 3}\right)$, we have $E \operatorname{size} e_{\min }(A)=\Omega\left(n^{5 / 3}\right)$ and Wbound $_{\min }(A)=\Omega\left(n^{8 / 3}\right)$. Again for 'good' three-dimensional meshes with $\lambda_{2}=\Theta\left(n^{-2 / 3}\right)$, we have $E_{\text {size }}$ min $(A)=\Omega\left(n^{4 / 3}\right)$ and $W$ bound $\min (A)=\Omega\left(n^{7 / 3}\right)$.

It is easy to establish that $\lambda_{n} \leq 2 \Delta$ for all graphs, by considering the eigenvalue equation $Q \underline{x}_{n}=\lambda_{n} \underline{x}_{n}$. Since we are considering bounded-degree finite element meshes, from 
Theorem 3.2, Theorem 5.2, and Theorem 2.1, the upper bound on the envelope size is Esize $(A)=\mathcal{O}\left(n^{2}\right)$ and that on the work bound is $W$ bound $(A)=\mathcal{O}\left(n^{3}\right)$. Notice that the gap between the lower bound and the upper bound on these parameters is quite large.

How good are the lower bounds on the envelope size and estimate of the envelope work? To answer this question, we prove upper bounds for these parameters from a "modified nested dissection" ordering of graphs with good separators. At each level, if a separator $S$ separates the current graph $G$ into two parts $A, B$, then in this ordering vertices in $A$ are numbered first, then the vertices in $S$, and finally the vertices in $B$. (See the ordering in Figure 2.1, where $S$ corresponds to the set of vertices in the middle column.) For simplicity of exposition, initially consider the class of planar graphs.

THEOREM 7.3. The minimum envelope size of a symmetric matrix of order $n, M$, whose adjacency graph $G$ is a bounded-degree planar graph, satisfies Esize $e_{\text {min }}(M)=\mathcal{O}\left(n^{1.5}\right)$.

Proof. Let a separator $S$ separate $G$ into parts $A$ and $B$, with $|A|=\alpha n \geq|B|=\beta n$, and $|S|=c \sqrt{n}$, where $\alpha+\beta=1$. From the planar separator theorem [26], $\alpha$ is bounded between $1 / 2$ and $2 / 3$. Consider the modified nested dissection ordering that numbers vertices in $A$ first, then vertices in $S$, and finally vertices in $B$. The contribution to the envelope size made by vertices in $S$ is at most $c \sqrt{n} \alpha n$, since each of them has row-width at most $|A|$. Also, there are at most $\Delta c \sqrt{n}$ vertices in $B$ adjacent to vertices in $S$; each of these vertices has row-width at most $\beta n$ since they may be numbered last in $B$, and hence such vertices contribute at most $c \sqrt{n} \Delta \beta n$ to the envelope size.

The reasoning above leads to the recurrence

$$
E(n) \leq E(\alpha n)+E(\beta n)+c(\alpha+\beta \Delta) n^{1.5} .
$$

Let $a=\alpha^{1.5}+\beta^{1.5}$. Then it is easy to show that $a<1$ since $\alpha+\beta=1$. The solution to the recurrence is

$$
E(n) \leq c(\alpha+\beta \Delta) \frac{a}{1-a} n^{1.5} .
$$

This completes the proof.

This technique can be used to prove that $W$ bound $(A)=\mathcal{O}\left(n^{2.5}\right)$ for matrices whose adjacency graphs are bounded-degree planar graphs. For matrices whose adjacency graphs (i) have bounded-degree, (ii) are embeddable in three dimensions, and (iii) possess $n^{2 / 3}$. separators, the upper bounds are $\operatorname{Esize}(A)=\mathcal{O}\left(n^{5 / 3}\right)$ and $W$ bound $(A)=\mathcal{O}\left(n^{8 / 3}\right)$. These results show that the lower bounds on the minimum envelope size (and the estimate of the work-bound) are asymptotically tight for two-dimensional meshes with $\lambda_{2}(G)=\Theta\left(n^{-1 / 2}\right)$, and for three-dimensional meshes with $\lambda_{2}(G)=\Theta\left(n^{-1 / 3}\right)$. Upper bounds as in the previous theorem can also be established for the class of bounded-degree overlap graphs embedded in $d$-dimensional space [28]; these graphs have $\mathcal{O}((d-1) / d)$-separators that separate them into two parts with at most $((d+1) /(d+2)) n$ vertices in the larger part.

The broader implication of the result above is that if a problem possesses good separators, then it has small envelope size and work. In practice, there exist orderings with smaller envelope parameters than the modified nested dissection ordering.

We conclude that for problems in dimensions greater than two, asymptotically envelope factorization schemes are not competitive with iterative methods or direct methods that 


\begin{tabular}{|c|c|c|c|c|c|}
\hline$|V|$ & $|E|$ & $\lambda_{2}$ & $\begin{array}{r}\text { Spectral } \\
\text { LB }\end{array}$ & $\begin{array}{r}\text { Spectral } \\
2 \text {-sum }\end{array}$ & $\operatorname{Gap}(\%)$ \\
\hline 18 & 48 & 2.00 & 969 & 978 & 0.9 \\
\hline 66 & 192 & $6.25 \mathrm{e}-1$ & $1.50 \mathrm{e}+4$ & $1.54 \mathrm{e}+4$ & 2.6 \\
\hline 258 & 768 & $1.65 \mathrm{e}-1$ & $2.36 \mathrm{e}+5$ & $2.53 \mathrm{e}+5$ & 6.9 \\
\hline 1,026 & 3,072 & $4.17 \mathrm{e}-2$ & $3.75 \mathrm{e}+6$ & $4.05 \mathrm{e}+6$ & 7.4 \\
\hline 4,098 & 12,270 & $1.05 \mathrm{e}-2$ & $6.00 \mathrm{e}+7$ & $6.44 \mathrm{e}+7$ & 7.3 \\
\hline 16,386 & 49,152 & $2.60 \mathrm{e}-3$ & $0.953 e+9$ & $1.03 e+9$ & 9.1 \\
\hline
\end{tabular}

TABLE 8.1

2-sums from the spectral reordering algorithm and lower bounds for triangulations of the sphere.

store and operate on only the nonzero elements. (For the latter, the storage requirements are $\mathcal{O}(n \log n)$ and work is $\mathcal{O}\left(n^{1.5}\right)$ for a two-dimensional problem; in three dimensions, these are $\mathcal{O}\left(n^{4 / 3} \log n\right)$ and $\mathcal{O}\left(n^{2}\right)$.) But when a two-dimensional mesh possesses a small second Laplacian eigenvalue, envelope methods may be expected to work well. Similar conclusions should hold for three-dimensional problems when the number of mesh-points along the third dimension is small relative to the number in the other two dimensions, and for two-dimensional surfaces embedded in three-dimensional space.

8. Computational results. We present computational results to verify how well the spectral ordering reduces the 2-sum. We report results on two sets of problems.

The first set of problems, shown in Table 8.1, is obtained from John Richardson's (Thinking Machines Corporation) program for triangulating the sphere. The spectral lower bounds reported are from Theorem 5.2. The results show that the spectral reordering algorithm computes values within a few percent of the optimal 2-sum, since the gap between the 2-sum lower bounds and the spectral 2-sums is within that range.

Table 8.2 contains the second set of problems, taken from the Boeing-Harwell and Nasa collections. Here the bounds are weaker than the bounds in Table 8.1. These problems have two features that distinguish then from the sphere problems. Many of them have less regular degree distributions-e.g., NASA1846 has maximum degree 41 and minimum degree 5 , though NACA is an exception. They also represent more complex geometries. Nevertheless, these results imply that the spectral 2-sum is within a factor of two of the -optimal value for these problems.

The gap between the computed 2-sums and the lower bounds could be further reduced in two ways. First, a local reordering algorithm applied to the ordering computed by the spectral algorithm might potentially decrease the 2-sum. Second, the lower bounds could be improved by incorporating diagonal perturbations to the Laplacian. We will consider both these issues in future work.

9. Conclusions. The lower bounds on the 2-sums show that the spectral reordering algorithm can yield nearly optimal values. To the best of our knowledge, these are the first results providing reasonable bounds on the quality of the orderings generated by a reordering algorithm for minimizing envelope-related parameters. Earlier work had not addressed the 


\begin{tabular}{rrr|r|rrr} 
Problem & $|V|$ & $|E|$ & $\lambda_{2}$ & $\begin{array}{r}\text { Spectral } \\
\text { LB }\end{array}$ & $\begin{array}{r}\text { Spectral } \\
2 \text {-sum }\end{array}$ & Gap(\%) \\
\hline CAN1072 & 1,072 & 5,686 & $7.96 \mathrm{e}-2$ & $8.17 \mathrm{e}+6$ & $9.02 \mathrm{e}+6$ & 9.4 \\
NASA1824 & 1,824 & 18,692 & $2.71 \mathrm{e}-1$ & $1.37 \mathrm{e}+8$ & $1.74 \mathrm{e}+8$ & 21 \\
NASA2146 & 2,146 & 35,052 & $1.35 \mathrm{e}-1$ & $1.11 \mathrm{e}+8$ & $1.32 \mathrm{e}+8$ & 16 \\
NACA & 4,224 & 12,416 & $3.57 \mathrm{e}-3$ & $2.24 \mathrm{e}+7$ & $2.70 \mathrm{e}+7$ & 17 \\
BARTH4 & 6,019 & 17,473 & $1.756-3$ & $3.19 \mathrm{e}+7$ & $5.41 \mathrm{e}+7$ & 41 \\
BARTH & 6,691 & 19,478 & $2.62 \mathrm{e}-3$ & $6.55 \mathrm{e}+7$ & $1.39 \mathrm{e}+8$ & 53
\end{tabular}

TABLE 8.2

2-sums from the spectral reordering algorithm and lower bounds for some problems from the BoeingHarwell and Nasa collections.

issue of the quality of the orderings generated by the algorithms. The lower bounds for the envelope size and envelope work are not useful in assessing the quality of envelope-reducing orderings, but are asymptotically tight for certain classes of 2- and 3-dimensional meshes. It remains to be seen if lower bounds obtained via the QAP formulation of the 1-sum problem lead to better results for the envelope size. Designing local reordering algorithms to improve the quality of the spectral orderings is another priority.

\section{REFERENCES}

[1] N. Alon and V. Milman, $\lambda_{1}$, isoperimetric inequalities for graphs, and superconcentrators, J. of Combin. Theory, Series B, 38 (1985), pp. $73-88$.

[2] S. T. Barnard, A. Pothen, and H. D. Simon, A spectral algorithm for envelope reduction of sparse matrices, Tech. Rep. CS-93-49, Computer Science, University of Waterloo, Oct. 1993. Submitted to J. Numerical Linear Algebra with Applications. A shorter version has appeared in the Proceedings of Supercomputing '93.

[3] E. H. CUthill and J. McKeE, Reducing the bandwidth of sparse symmetric matrices, in Proceedings of the 24th Nat. Conf. ACM, ACM Publications, 1969, pp. 157-172.

[4] E. F. D'Azevedo, P. A. Forsyth, AND W. P. TANG, Ordering methods for preconditioned conjugate gradients methods applied to unstructured grid problems, SIAM J. Matrix Anal. Appl., 13 (1992), pp. 944-961.

[5] I. S. Duff, A. M. ERisman, And J. K. Reid, Direct Methods for Sparse Mătrices, Clarendon Press, Oxford, 1986.

[6] I. S. DUfF AND G. A. MEURANT, The effect of ordering on preconditioned conjugate gradients, BIT, 29 (1989), pp. $635-657$.

[7] I. S. DUFF, J. K. REID, AND J. A. ScotT, The use of profile reduction algorithms with a frontal code, International Journal for Numerical Methods in Engineering, 28 (1989), pp. 2555-2568.

[8] M. FIEDLER, Algebraic connectivity of graphs, Czech. Math. J., 23 (1973), pp. 298-305.

[9] - A property of eigenvectors of non-negative symmetric matrices and its application to graph theory, Czech. Math. J., 25 (1975), pp. 619-633.

[10] G. Finke, R. F. Burkard, and F. Rend, Quadratic assignment problems, in Surveys in Combinatorial Optimization, S. Martell et al., ed., vol. 31 of Annals of Discrete Mathematics, Elsevier Science Publishers, 1987, pp. 61-82.

[11] N. GafFKe and O. KRAFFT, Matrix inequalities in the Löwner orderings, in Modern Applied Mathematics: Optimization and Operations Research, B. Korte, ed., North Holland, 1982, pp. 576-622.

[12] A. George, Computer implementation of the finite element method, Tech. Rep. 208, Department of 
Computer Science, Stanford University, Stanford, CA, 1971.

[13] A. George and J. W. H. Liv, Computer Solution of Large Sparse Positive Definite Systems, Prentice Hall, 1981.

[14] N. E. GiBBS, Algorithm 509: A hybrid profile reduction algorithm, ACM Trans. on Math. Software, 2 (1976), pp. 378-387.

[15] N. E. Gibbs, W. G. Poole, JR., And P. K. Stockmeyer, An algorithm for reducing the bandwidth and profile of a sparse matrix, SIAM J. Num. Anal., 13 (1976), pp. $236-249$.

[16] R. G. Grimes, D. J. Pierce, and H. D. Simon, A new algorithm for finding a pseudoperipheral node in a graph, SIAM J. Matrix Anal. Appl., 11 (1990), pp. $323-334$.

[17] S. HADLEY, F. RENDL, AND H. WolkowiCz, A new lower bound via projection for the quadratic assignment problem, Mathematics of Operations Research, 17 (1992), pp. 727-739.

[18] C. Helmberg, B. Mohar, S. PoljaK, ANd F. Rendl, A spectral approach to bandwidth and separator problems in graphs. Preprint, Department of Mathematics, University of Ljubljana, Jadranska 19, 61 111, Ljubljana, Slovenia, Feb. 1993.

[19] B. A. HENDRICKSON AND R. W. LELAND, An improved spectral graph partitioning algorithm for mapping parallel computations, Tech. Rep. 92-1460, Sandia National Labs, Albuquerque NM, 1992.

[20] _-, Multidimensional load balancing, Tech. Rep. 93-0074, Sandia National Labs, Albuquerque NM, 1993.

[21] M. JUVAN AND B. MoHAR, Laplace eigenvalues and bandwidth-type invariants of graphs. Preprint, Department of Mathematics, University of Ljubljana, Jadranska 19,61 111, Ljubljana, Slovenia, 1990.

[22] —- Optimal linear labelings and eigenvalues of graphs, Discr. Appl. Math., 36 (1992), pp. 153-168.

[23] J. G. LEwIS, Implementations of the Gibbs-Poole-Stockmeyer and Gibbs-King algorithms, ACM Trans. on Math. Soft., 8 (1982), pp. $180-189$.

[24] Y. LIN AND J. YUAN, Minimum profile of grid networks in structure analysis. Preprint, Department of Mathematics, Zhengzhou University, Zhengzhou, Henan 450052, People's Republic of China, 1993.

[25] — Profile minimization problem for matrices and graphs. Preprint, Department of Mathematics, Zhengzhou University, Zhengzhou, Henan 450052, People's Republic of China, 1993.

[26] R. J. Lipton and R. E. TARJan, A separator theorem for planar graphs, SIAM J. Appl. Math., 36 (1979), pp. 177-189.

[27] J. W. H. LiU And A. H. Sherman, Comparative analysis of the Cuthill-Mckee and the reverse CuthillMckee ordering algorithms for sparse matrices, SIAM J. Numer. Anal., 13 (1976), pp. 198-213.

[28] G. L. Miller, S.-H. Teng, W. Thurston, and S. A. Vavasis, Automatic mesh partitioning, in Graph Theory and Sparse Matrix Computation, A. George, J. R. Gilbert, and J. W. H. Liu, eds., Springer Verlag, 1993, pp. 57-84. The IMA Volumes in Mathematics and its Applications, Volume 56 .

[29] B. Mohar and S. PoljaK, Eigenvalues in combinatorial optimization. Preprint, Department of Mathematics, University of Ljubljana, Jadranska 19,61 111, Ljubljana, Slovenia, 1992.

[30] G. H. Paulino, I. F. M. Menezes, M. Gattass, and S. Mukherjee, Node and element resequencing using the Laplacian of a finite element graph, Parts I and II. Preprint, School of Civil and Environmental Engineering, Cornell University, 1993. To appear in International Journal for Numerical Methods in Engineering.

[31] A. Pothen, H. D. Simon, AND K. P. LIOU, Partitioning sparse matrices with eigenvectors of graphs, SIAM J. Matrix Anal. Appl., 11 (1990), pp. 430-452.

[32] A. Pothen, H. D. Simon, And L. Wang, Spectral nested dissection, Tech. Rep. CS-92-01, Computer Science, Pennsylvania State University, University Park, PA, 1992. A shorter version has appeared in the Proceedings of Supercomputing ' 92.

[33] A. Pothen AND L. WANG, An improved spectral nested dissection algorithm. In preparation, 1994.

[34] F. RENDl AND H. Wolkowicz, A projection technique for partitioning the nodes of a graph. To appear in a special issue of Annals of Operations Research devoted to the Symposium on Applied Mathematical Programming and Modeling, Budapest, Jan. 1993. (This paper was written in 1990.)

[35] H. D. Simon, Partitioning of unstructured problems for parallel processing, Computing Systems in Engineering, 2 (1991), pp. 135-148. 
[36] S. W. Slóan, An algorithm for profile and wavefront reduction of sparse matrices, International Journal for Numerical Methods in Engineering, 23 (1986), pp. 239-251.

\section{Appendix}

A. Lower bounds on the minimum $p$-sum. We prove two lower bounds on the minimum $p$-sums. We make use of Lemma 3.1 in proving the first result. In the following $B_{m}(x)$ is the $m$ th Bernoulli polynomial, and $B_{m}$ is the $m$ th Bernoulli number.

THEOREM A.1. For $1 \leq p<\infty$, the minimum $p$-sum of a graph $G$ on $n$ vertices satisfies

$$
\sigma_{p}^{p} \geq \frac{1}{p+1}\left(B_{p+1}(s+1)-B_{p+1}\right)
$$

where $s=\left(\lambda_{2} / 4 \Delta\right) n$.

Proof. Consider any ordering $\alpha$ of the vertices of $G$. Partition the vertices into two sets: $A$ consisting of the lowest-numbered $n / 2$ vertices, and $B$ consisting of the highest-numbered $n / 2$ vertices. By Lemma 3.1 the number of edges joining $A$ and $B,|\delta(A, B)|$, is

$$
|\delta(A, B)| \geq \frac{\lambda_{2}}{n}(n / 2)^{2} .
$$

Hence at least $s=|\delta(A, B)| / \Delta$ vertices in $B$ are adjacent to vertices in $A$. Each vertex in this subset of $B$ has the least row-width when it is adjacent to the highest-numbered vertex in $A$ and to no other vertices in $A$. Hence these $s$ vertices make a contribution of at least $1^{p}+\ldots+s^{p}$ to the $p$-sum, and this sum can be expressed in terms of the Bernoulli polynomials as stated.

From an expansion of the Bernoulli polynomial, we find that asymptotically

$$
\sigma_{p}^{p} \geq \frac{1}{(p+1)(4 \Delta)^{p+1}} \lambda_{2}{ }^{p+1} n^{p+1}+\mathcal{O}\left(\left(\lambda_{2}{ }^{p} / \Delta^{p}\right) n^{p}\right) .
$$

We proceed to obtain another lower bound on the minimum $p$-sum.

The next result makes use of the following Lemma A.2 recently proved by Helmberg et al. [18]. Define the following symmetric function of the two positive integers $m_{1}, m_{2}$ (with $\left.m_{1}+m_{2}<n\right)$ and parameters $\lambda_{2}, \lambda_{n}$ :

$$
\begin{aligned}
& \text { (A.1) } f\left(m_{1}, m_{2}\right)= \\
& \frac{\sqrt{m_{1} m_{2}}}{2 n}\left[\left(\sqrt{m_{1} m_{2}}+\sqrt{\left(n-m_{1}\right)\left(n-m_{2}\right)}\right) \lambda_{2}+\left(\sqrt{m_{1} m_{2}}-\sqrt{\left(n-m_{1}\right)\left(n-m_{2}\right)}\right) \lambda_{n}\right] .
\end{aligned}
$$

Lemma A.2. Let $S_{1}, S_{2}$ be two disjoint subsets of the vertices of a graph $G$ on $n$ vertices, with $\left|S_{i}\right|=s_{i}$, for $i=1,2$. Then the number of edges joining $S_{1}$ and $S_{2},\left|\delta\left(S_{1}, S_{2}\right)\right|$, satisfies

$$
\left|\delta\left(S_{1}, S_{2}\right)\right| \geq f\left(s_{1}, s_{2}\right) .
$$


TheOrem A.3. For $1 \leq p<\infty$, the minimum $p$-sum of a graph $G$ satisfies

$$
\sigma_{p}^{p} \geq \frac{1}{2^{p+1} \Delta} \frac{\lambda_{2}^{p+1}}{\left(\lambda_{n}+\lambda_{2}\right)^{p+2}}\left(2 \lambda_{n}+\lambda_{2}\right)\left(\lambda_{n}+2 \lambda_{2}\right) n^{p+1} .
$$

Proof. Consider any ordering $\alpha$ of the vertices of $G$, and consider a tripartition $A, B$, $C$ : We choose $A$ to consist of the lowest-numbered $a \equiv(n-b) / 2$ vertices, $C$ to consist of the highest-numbered $(n-b) / 2$ vertices, and $B$ to contain the remaining $b$ vertices in the 'middle'. Here $b$, the size of $B$, is a parameter that will be determined later to obtain a large lower bound.

From Lemma A.2, $|\delta(A, C)|$, the number of edges joining $A$ and $C$, is at least $f(a, a)$, where the symmetric function $f(.,$.$) is defined in (A.1). Hence there are at least s_{C}=$ $f(a, a) / \Delta$ vertices in $C$ adjacent to vertices in $A$. Each of these vertices has row-width at least $b$.

Initially, consider the contribution to the envelope size $\operatorname{Esize}(G)$ made by these vertices to obtain a suitable value for $b$.

$$
\begin{aligned}
\operatorname{Esize}(G) & \geq \frac{f(a, a)}{\Delta} b \\
& =\frac{(n-b)}{4 n}\left[\left(\frac{n-b}{2}+\frac{n+b}{2}\right) \lambda_{2}+\left(\frac{n-b}{2}-\frac{n+b}{2}\right) \lambda_{n}\right] \frac{b}{\Delta} \\
& =\frac{1}{4 \Delta} b(n-b)\left(\lambda_{2}-(b / n) \lambda_{n}\right) .
\end{aligned}
$$

We choose $b$ to maximize the lower bound on the envelope size. Differentiating the cubic polynomial in (A.2) with respect to $b$ and simplifying, we obtain the quadratic equation

$$
b^{2}-\frac{2}{3} \frac{\lambda_{2}+\lambda_{n}}{\lambda_{n}} n b+\frac{1}{3} \frac{\lambda_{2}}{\lambda_{n}} n^{2}=0 .
$$

From the quadratic we find that the maximizer is, to first order, $b_{m}=(1 / 2)\left(\lambda_{2} /\left(\lambda_{n}+\lambda_{2}\right)\right) n$.

Now we consider the contribution to the $p$-sum made by the $s_{C}$ vertices in $C$ adjacent to vertices in $A$. Each of these vertices contributes at least $b^{p}$ to the $p$-sum, and thus a lower bound on the minimum $p$-sum is

$$
\sigma_{p}^{p}(G) \geq \frac{1}{4 \Delta}(n-b)\left(\lambda_{2}-(b / n) \lambda_{n}\right) b^{p} .
$$

It is not easy to find a maximizer of the right-hand-side in the bound above on the $p$-sum since the polynomial in $b$ is of degree $p+2$. Hence we choose $b$ equal to the maximizer of the envelope size. We obtain the bound stated in theorem by substituting $b=b_{m}$ in the bound above.

Juvan and Mohar [22] have proved upper bounds for the $p$-sums. The techniques in this Appendix can be used to compute bounds on $\operatorname{Esize}(A)$ and $W$ bound $(A)$, but the results are weaker than those obtained in Section 3. 
Public reporting burden for this collection of information is estimated to average 1 hour per response, including the time for reviewing instructions, searching existing data sources. gathering and maintaining the data needed, and completing and reviewing the collection of information. Send comments regarding this burden estimate or any other aspect of this Davis Highway, Suite 1204, Arlington, VA 22202-4302, and to the Office of Management and Budget. Paperwork Reduclion Project (0704 0188). Washington, DC 20503.

\begin{tabular}{|l|l|l} 
1. AGENCY USE ONLY(Leave blank) & $\begin{array}{c}\text { 2. REPORT DATE } \\
\text { October 1994 }\end{array}$ & $\begin{array}{l}\text { 3. REPORT TYPE AND DATES COVERED } \\
\text { Contractor Report }\end{array}$ \\
\hline
\end{tabular}

4. TITLE AND SUBTITLE

AN ANALYSIS OF SPECTRAL ENVELOPE-REDUCTION VIA QUADRATIC ASSIGNMENT PROBLEMS

C NAS1-19480

WU 505-90-52-01

6. AUTHOR(S)

Alan George

Alex Pothen

7. PERFORMING ORGANIZATION NAME(S) AND ADDRESS(ES)

Institute for Computer Applications in Science

and Engineering

Mail Stop 132C, NASA Langley Research Center

5. FUNDING NUMBERS

Hampton, VA 23681-0001

9. SPONSORING/MONITORING AGENCY NAME(S) AND ADDRESS(ES)

National Aeronautics and Space Administration

Langley Research Center

Hampton, VA 23681-0001

8. PERFORMING ORGANIZATION

REPORT NUMBER

ICASE Report No. $94-81$

10. SPONSORING/MONITORING AGENCY REPORT NUMBER

NASA CR-194992

ICASE Report No. 94-81

\section{SUPPLEMENTARY NOTES}

Langley Technical Monitor: Michael F. Card

Final Report

Submitted to SIAM Journal on Matrix Analysis and Its Applications, September 1994

12a. DISTRIBUTION/AVAILABILITY STATEMENT

12b. DISTRIBUTION CODE

Unclassified-Unlimited

Subject Category 64

13. ABSTRACT (Maximum 200 words)

A new spectral algorithm for reordering a sparse symmetric matrix to reduce its envelope size was described in [2] The ordering is computed by associating a Laplacian matrix with the given matrix and then sorting the components of a specified eigenvector of the Laplacian. In this paper, we provide an analysis of the spectral envelope reduction algorithm. We described related 1- and 2-sum problems; the former is related to the envelope size, while the latter is related to an upper bound on the work involved in an envelope Cholesky factorization scheme. We formulate the latter two problems as quadratic assignment problems, and then study the 2 -sum problem in more detail. We obtain lower bounds on the 2-sum by considering a projected quadratic assignment problem, and then show that finding a permutation matrix closest to an orthogonal matrix attaining one of the lower bounds justifies the spectral envelope reduction algorithm. The lower bound on the 2 -sum is seen to be tight for reasonably "uniform" finite element meshes. We also obtain asymptotically tight lower bounds for the envelope size for certain classes of meshes.

\section{SUBJECT TERMS}

I-sum problems, 2-sum problems, envelope reduction, eigenvalues of graphs, Laplacian matrices, quadratic assignment problems, reordering algorithms, sparse matrices
15. NUMBER OF PAGES 25

16. PRICE CODE $\mathrm{A} 03$

17. SECURITY CLASSIFICATION OF REPORT

Unclassified

18. SECURITY CLASSIFICATION OF THIS PAGE

Unclassified

19. SECURITY CLASSIFICATION OF ABSTRACT 
$\longrightarrow$ 


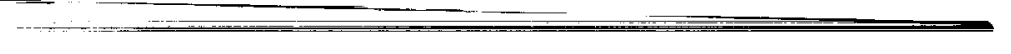

烈 